\title{
Role of Human Factor and Non Technical Skills in Anaesthetic Nursing Practice: A Case Study
}

\author{
Martin $\mathrm{D}^{1 *}$, MeLarkey $\mathrm{N}^{2}$ and Hoy $\mathrm{L}^{3}$ \\ 1Pathwy Leader Specialist Practice in Anaesthetic Nursing, Queen's University \\ Belfast, N. Ireland \\ ${ }^{2}$ Anaesthetic Nurse Specialist, South West Acute Hospital, N. Ireland \\ ${ }^{3}$ Director Specialist Practice in Nursing, Queen's University Belfast, N. Ireland
}

\section{Case Report \\ Volume 3 Issue 1}

Received Date: November 28, 2017

Published Date: January 19, 2018

*Corresponding author: Daphne Martin, Lecturer (Education), Pathwy Leader Specialist Practice in Anaesthetic Nursing, Queen's University Belfast, N. Ireland, Tel: 02890972389; E-mail: d.s.martin@qub.ac.uk

\section{Abstract}

The role of the anaesthetic nurse specialist is about change and adaptation, consisting of many skills including educator, advisor, change agent and innovator. Expertise, knowledge and skills acquired, range not only from years of experience, but now through a more formal educational specialist pathway at graduate level. The anaesthetic specialized role is to enhance patient outcomes and experience by delivering individually tailored care in the Perioperative phase. The need to improve the quality of patient care and reform the NHS into an organization fit for the future has been the focus of many debates in national government, but in the end, it all comes down to efficacy. Effectiveness, value for money and productivity are the drivers in the development of many new specialized roles, especially in the Perioperative environment. Staff development and education is vital, nevertheless, in these current times of budget cuts and austerity measures continuing professional development can be sacrificed. Therefore as a specialist practice nurses, we must be innovative in finding ways in improving our practice and developing our knowledge and skills.

Keywords: Specialist Anaesthetic Nurse; laryngospasm; Reflective practice; Human factors; Non-technical skills

Abbreviations: ANS: Anaesthetic Nurse Specialist; PACU: Post Anaesthetic Care Unit; DPU: Day Procedure Unit; ETT: Endo Tracheal Tube; NMC: Nursing and Midwifery Council.

\section{Introduction}

Critical incident analysis is a fundamental tool used for improving patient safety, and contributes towards the provision of high quality of care [1-5]. Flanagan's archive work from 1954 described how analysis of critical incidents can be used to determine the facts, find solutions and learn from mistakes. It can help minimize the risk of similar events occurring again, whilst allowing valuable lessons to be learnt in the clinical setting. Critical incidents do happen unfortunately, in the peri-operative arena, where there is always an element of risk to the public when they are in a vulnerable state [1] and in need of our services. Kirby [6] acknowledges critical incidents as events that can inflict injury and loss that may arise 


\section{Anaesthesia \& Critical Care Medicine Journal}

from human error, negligence or a series of complexities. Patients are most vulnerable when in hospital and it is our duty to safeguard and protect them [7]. Critical incidents can have both a negative and positive impact on staff. They can awaken various feelings, sometimes distressing memories for the team involved depending on the event. Reflecting on the incident can have many benefits, acting as a learning tool, to improve our practice, act as a debriefing of what occurred and constructs our own knowledge, skills and abilities by closely examining our actions or omissions. In critical incident analysis, reflection plays a vital role. To learn from our experiences we must reflect using a process that will identify the event and draw out why it was of particular significance to us, and hope to gain greater insight and awareness of it, hence reducing risks of any untoward event happening again [8]. There are several different approaches to reflection, for example Gibb's Reflective Cycle Model (1988), Mezirows Levels of Reflectivity (1991), Johns Model of Structured Reflection (2002) and the Scottish Executive Critical Incident Framework (2003). Gibb's Reflective Cycle Model (1988) will be used here to form a structured analysis of a clinical incident. Gibb's uses description, thoughts and feelings, evaluation, analysis, conclusion and action planning [9] as a means of exploring the experience whilst increasing knowledge and problem solving [10].

\section{Description}

The first steps in Gibb's G [11] reflective cycle relates to a description of the incident allowing the practitioner to look back on events. The incident occurred at the end of a busy paediatric dental list, in a Day Procedure Unit (DPU) of a small undergraduate teaching hospital. We had our team brief/ huddle [12] prior to commencing the list, with theatre staff, anaesthetist, dental team and DPU nursing staff. The Anaesthetic Nurse Specialist (ANS) delegated the duties for the list. A joint decision was made between the ANS and senior nurse to take it in turn to perform the anaesthetic and Post Anaesthetic Care Unit (PACU) nurse role of each child, and then follow them through. This was decided at the team brief, there was no dedicated PACU nurse assigned to the patient operating list.

\section{Case Study}

The child was a fit and healthy eight year old boy admitted as a day case for five dental extractions under general anaesthetic. He was $29 \mathrm{~kg}$ with no allergies, fasted for six hours as per hospital policy and AAGBI guidelines, had no medical or surgical history, and no previous anesthetics. His mother was informed of the procedure and signed consent attained. He was given pre-medication of Paracetamol and Ibuprofen analgesia; he arrived at theatre in his own clothes with his mother and teddy bear. There was music playing in the background, which can help relieve patient's anxiety. The child was relaxed in the situation and his mother kept him informed of what was happening. Standard monitors (pulse oximetry, blood pressure, electrocardiograph) where placed and he was cannulated without concern. He was preoxygenated for three minutes, given $30 \mathrm{mcg}$ of Fentanyl intravenously (IV), gas induction of Sevoflurane tolerated well. This was followed by administration of Suxamethonium, a muscle relaxant, for its short acting properties and intubated under direct laryngoscopy with a size $6 \mathrm{~mm}$ micro cuff endotracheal tube (ETT). At the first attempt correct placement was confirmed by end tidal $\mathrm{CO}_{2}$, bilateral chest sounds and equal chest excursion. Intra-operative WHO safety check list was completed with no issues raised, anaesthesia was maintained with sevoflurane and the surgical team successfully completed the five dental extractions. Monitoring indicated no anomalies and within 15 minutes the child was ready to be wakened. On waking the child, after extubation of the ETT, he took some spontaneous breathes. A non-rebreather oxygen mask was placed and $0_{2}$ administered at $5 \mathrm{~L}$ per minute, monitors where removed and we were ready to transfer to the PACU. However, very quickly his breathing slowed, and in a noisy theatre, a high pitched wheeze was heard on inspiration resembling a stridor. The anesthetist suggested the child had laryngospasm. He asked for the facemask and Guedal airway that we had previously used but they had been discarded, as well as the anaesthetic tubing from the anaesthetic machine. The ANS quickly fetched the required items and asked for another nurse to assist with reattaching the tubing and monitors whilst the ANS prepared a new ETT. The anesthetist was finding it difficult to ventilate therefore the ANS assisted in a two person technique but this was not ending the laryngospasm. The child's $\mathrm{SaO}_{2}$ was now $64 \%$ and visibly cyanosed. Help was audibly summoned for another nurse to put out a critical care call for further anaesthetic assistance and a second nurse sent for the emergency drug box along with Propofol and Rocuronium that the anesthetist had asked for. The drug cupboards were locked! However teamwork prevailed and within 60 seconds the drugs were administered, hand ventilation was successful and the child was reintubated. We remained in the theatre for one and a half hours, until the effects of the neuromuscular block had worn off as indicated by the train-of-four wave form on the nerve stimulator. The child was successfully extubated when fully awake and then transferred to the PACU where the ANS monitored him for a further hour without any 


\section{Anaesthesia \& Critical Care Medicine Journal}

complications, other than a sore throat. He was moved to an in-patient bed in the children's ward for overnight observation.

\section{Thoughts and Feelings}

At the beginning of the list, during the brief, the ANS was relaxed commencing the list. We have two regular paediatric lists per week and were prepared for our theatre list. There was a feel good mood in the theatre, atmosphere being pleasant and friendly. The mood soon turned very sombre, with the development of this child's laryngospasm. The ANS quickly realized the seriousness of the situation, felt scared and concerned for the child. However, as team lead the ANS was aware for rapid management of the situation, instructing the team, what action was necessary. Bostrom, et al. [13] suggests where a specialist nurse practitioner is seen to handle an emergency situation it gives colleagues a sense of security and safety in their role. The ANS felt she communicated clearly the plan of action and instructions were concise, easy to follow and remained positive. Nevertheless, following the critical event, the ANS was emotional and tearful at home. Reflecting on the sequence of events the ANS considered that as a team we had let the patient down. She had become distracted although it wouldn't have prevented the event from occurring but her personal reaction may have been quicker. The ANS considered the "what ifs", felt ill at the thought of what could have happened! A change of behavior was necessary to ensure patient safety and prevent an occurrence of this situation again. Professionalism in the theatre team was relateable as Smith [14] suggests changing cultures of complacency, in order to eliminate errors in the future.

\section{Evaluation}

Gibbs G [11] reflective model recommends evaluation of the event, in order to appraise both positive and negative outcomes. The positive upshot of this incident was the child made a full recovery with no ill effects and we managed an unanticipated difficult airway successfully. Undoubtedly, the difficulties faced where more the result of human factors connected to staffing, distraction, lack of concentration and complacency. These factors contributed to the patient safety leaving them even more vulnerable and their Perioperative care not adequately patient focused [11].

The AAGBI [15], suggests that the anaesthetic nurse has no other roles or duties to fulfil other than providing knowledgeable and skilled anaesthetic assistance. In this incident, we did not go with these recommendations. The
Post Anaesthetic Care Unit (PACU) staff shortage had been discussed at the briefing session prior to commencing the list, but as a team we had chosen to continue on without delay, to avoid cancelling any patients. We already had problems with staffing a dedicated PACU, but instead of seeking further advice or additional support from another department we continued on as we always did. Hindsight would suggest the ANS should have brought this matter to the departmental manager. The NHS has seen the impact of government austerity measures, with staffing shortages of major concern to most hospital trusts. Nevertheless, as ANS we are still held accountable by the NMC [7] to act as advocate and safeguard each patient in our care.

A team brief or "huddle" is an opportunity for the team to raise concerns regarding each individual patient on the operating list, giving everyone an equal voice. It addresses operational safety issues whilst establishing the team concept and maintaining effective communication [16]. This is additional to the WHO (2010) surgical safety checklist that is used in the theatre department as opposed to the ward in this instance [17] wrote a letter in reply to an article regarding the preoperative safety checklist, adding that we may lose sight of the importance and lack concentration due to routine of the questions, suggesting that we may get complacent. Although this scenario was unanticipated and followed an otherwise uneventful case and list, the ANS wondered if it was possible that we hastily dismissed both the morning brief and pre-operative checklist (identifying shortage of staff for PACU).

The ANS was mindful that staff had started to clean and clear up the theatre prior to the child's first attempt of transfer to the PACU, yet she did not ask them to stop!. On reflection the ANS did not want to upset the good vibe by appearing bossy or domineering. Gardezi, et al. [18] conducted a study into staff feeling constrained in the operating room for fear of being misinterpreted, remaining silent when they know they should have spoken out. As team leader the ANS should have been more assertive, needless to say now, the ground rules, culture and traditions have since changed on the back of this incident and the entire theatre suites in the department do not: lock cupboards, start cleaning or disposing of any equipment, until the patient has safely entered the PACU.

\section{Analysis}

Gibb's G [11] identifies the next step in the reflective model as analysis. Distractions in the operating room 


\section{Anaesthesia \& Critical Care Medicine Journal}

(OR) are cited in numerous studies as having an impact on patient safety. Allen [19] identified several predominate factors including, telephones, bleeps, staff entering and exiting the operating room, teaching staff and students, case irrelevant conversations and music. In this incident, all of the above has led to complacency issues and events that put the child in danger. However telephones, bleepers, teaching all have a place within OR, the inappropriate conversations, along with the music do not. Its lovely having a relaxed working environment where there is good team relationships, but not to the detriment of patient safety. Evidence has shown that music in the OR can have mood enhancing effect on both patients and staff, but it can impair communication, interfere with the rhythmic signals of monitors, increases noise limits which in turn decrease our active listening, rising to attention/ concentration problems which affects our performance.

The world of aviation with its emphasis on safety has directly influenced our current approach to anaesthesia safety and healthcare in general. Many lessons learned from human factors, safety systems, checklists and improved training make the patients journey through anaesthesia, surgery and recovery what we expect for each individual patient that is uneventful [20]. Human factors address individual behaviours by raising awareness of how our abilities, limitations and relationship to the work environment can affect performance and safety. Similarly to aviation, Broom, et al. [21] listed three critical phases of general anaesthesia. Induction, maintenance and emergence as opposed to take off, cruise and landing. The induction phase should be calm, quiet and free from interruption and distraction. Full concentration from the anaesthetic team is required for the surgery and airway. This is known as the sterile anaesthetic cockpit concept [21]. This includes 100\% focus on the important elements in delivery of the anaesthetic (patient, the anaesthetic team, and the drugs and equipment). The emergence phase or awakening of the patient, should be treated with the same consideration, however it tends to be nosier and busier. This can lead to adverse incidents occurring through distraction and confusion. Evidently in this scenario team concentration had slipped during this phase, the noise level had increased with more theatre personnel in the vicinity, as well as equipment movement and bins rattling. These factors, as well as others, were outlined in Broom, et al. [21] as having a direct impact on the anaesthetic team's situational awareness and responsiveness to emergencies. Situational awareness is being perceptive of our environment through our knowledge experience and skill. It enables us to understand what is happening so we can determine if it is safe. Detecting, integrating and interpreting the data we gather from our environment, then communicating the processed information to our colleagues on how to react. It is thought to be one of the most essential non-technical skills for safe anaesthetic practice [20]. However the concept of situational awareness is unique to each individual, what one considers dangerous another may not, hence making it arbitrary [22-26].

\section{Conclusion}

In conclusion, the child suffering from laryngospasm, a critical event that required prompt action and management from a specialized team. Our focus is always doing good for patients and reducing risks. The child came to no harm, however reflecting on the incident several areas of concern that could have delayed our reaction time, potentially putting the child at risk were identified. We may not have been able to prevent the laryngospasm, but the delayed few minutes in a difficult airway event can be catastrophic, having life changing effects on the patient, if not death.

Following this incident, several changes have occurred that will promote a safer environment for every theatre user with a clear focus on the patient. Culture, traditions and practice were examined. The ANS has introduced a sterile anaesthetic cockpit where theatre personnel keep to minimum noise levels during the induction and emergence of anaesthesia, music is now switched off during these times. The ANS felt the need to reinforce some of the ground rules in theatre to address complacency. Clearing and cleaning of theatre does not happen until the patient has exited the theatre room and no drug cupboards are locked whilst a patient is present. These are very basic changes but it was a shock to find how these simple practices crept in unnoticed to the team.

The ANS created a new paediatric difficult airway trolley in collaboration with the paediatric lead anaesthetist; it has been introduced into the paediatric ward, the intensive care unit (ICU) and emergency department (ED). We have been able to secure teaching for paediatric airway management and use of the trolley as well as working on skills stations for scenario based training in such emergency events.

The biggest change for the ANS was a change of role to that of Theatre Sister. A developing and extending role has challenged opinion, practice and skills. Daily the ANS is engaged with many different patient scenarios, this has driven the need to improve knowledge, understanding 


\section{Anaesthesia \& Critical Care Medicine Journal}

and expertise bedsides a two-year Nursing and Midwifery Council (NMC) approved Post Graduate Specialist Practice Anaesthetic Nursing programme at Queen's University Belfast, School of Nursing and Midwifery. Critical incident analysis grows an awareness of our behavior, abilities and permits a challenge to current practices. Making positive changes if they are required to ensure patient safety and high quality patient care remains paramount. The role of the specialist anaesthetic nurse is to promote excellence in the field. We possess unique knowledge, skill and competencies that enable us to develop and nurture critical thinking in the team. Reflection provides a wealth of learning opportunities that can strengthen our understanding and influence future practice.

\section{References}

1. Cousley A, Martin D, Hoy L (2014) Vulnerability in the Perioperative Patient: A Concept Analysis. Journal of Perioperative Practice 24(7-8): 164-171.

2. Wickham S (2013) what are the Roles of Clinical Nurses and Midwife Specialists? British Journal of Nursing 22(15): 867-875.

3. Abraham J (2011) Innovative Perioperative Role Improves Patient and Organisational Outcomes in Minimal Invasive Surgery. Journal of Perioperative Practice 21 (5): 158-164.

4. Department of Health (2010) Quality, Innovation, Productivity and Prevention. London: DH.

5. Smith AF, Mahajan RP (2009) National Critical Incident Reporting: Improving Patient Safety. British Journal of Anaesthesia 103(5): 623-625.

6. Kirby EA (2011) A Conceptual Model for Critical Incident Analysis. Journal of Critical Incident Analysis 1(1).

7. Nursing and Midwifery Council (NMC) (2015) Code of Conduct: Standards of Conduct, Performance and Ethics for Nurses and Midwives. London: NMC.

8. Vachon B, LeBlanc J (2011) Effectiveness of Past and Current Critical Incident Analysis on Reflective Learning and Practice Change. Medical Education 45(9): 894-904.

9. Lewis G (2015) A Critical Reflection into a Perioperative Death and the Use of the WHO Safer Surgery Checklist Team Briefing and Debriefing using Gibb's Reflective Cycle. The Operating Theatre Journal (298): 14-15.

10. Jasper M, Rosser M, Mooney G (2013) Professional Development, Reflection and Decision-Making in Nursing and Healthcare. $2^{\text {nd }}(E d n$.$) , West Sussex.$ Wiley-Blackwell.

11. Gibbs G (1988) Learning by Doing: A Guide to Teaching and Learning Methods. Oxford Oxford Polytechnic, Further Education Unit.

12. Agency for Healthcare and Quality (AHRQ) (2014) Clarifying Key Concepts and Tools to Enhance Performance and Strategy.

13. Bostrum M, Magusson K, Engstrom A (2012) Nursing Patients Suffering from Trauma: Critical Care Nurses Narrate their Experiences. International Journal of Orthopaedic and Trauma Nursing 16: 21-29.

14. Smith M (2013) How to Diagnose Complacency: It Can Be a Fatal Condition! EMS World 42(4): 18.

15. Association of Anaesthetists of Great Britain and Ireland (2010) the Anaesthetic Team. London: AAGBI.

16. Powell SM, Hill RK (2006) My Co-Pilot is a Nurse Using Crew Resource Management in the OR. Association of Operating Room Nurses Journal 83 (1): 179-180.

17. Moss C (2013) Beware of Complacency in PreOperative Safety Checklists. Journal of Operating Practice 4(2): 17.

18. Gardezi F, Lingard L, Epsin S, Whyte S, Crser B, et al. (2009) Silence, Power and Communication in the Operating Room. Journal of Advanced Nursing 65(7): 1390-1399.

19. Allen G (2014) Evidence Appraisal of Antonidis et al. Identification and Interference of Intra-Operative Distractions and Interruptions in Operating Rooms. Journal of Association of Operating Room Practitioners 100 (3): 340-344.

20. Toff NJ (2010) Human Factors in Anaesthesia: Lessons from Aviation. British Journal of Anaesthesia 105 (1): 21-25. 


\section{Anaesthesia \& Critical Care Medicine Journal}

21. Broom MA, Capek AL, Carachi P, Akeroyd MA, Hilditch G (2011) Critical Phase Distractions in Anaesthesia and the Sterile Cockpit Concept. Anaesthesia 66(3): 175-179.

22. Flanagan JC (1954) the Critical Incident Technique. Psychological Bulletin 51 (4): 327-358.

23. Johns C (2009) Guided Reflection, Advancing Practice. London: Blackwell Science.
24. Kneebone R, Darzi A (2005) New Professional Roles within Surgery. British Medical Journal 330 (7495): 803-804.

25. Mezirow J (1991) Fostering Critical Reflection in Adulthood. San Francisco. California, USA: JosseyBass.

26. Scottish Executive (2003) the Framework for Social Work Education in Scotland. Edinburgh: The Stationary Office.

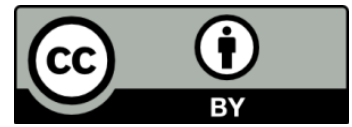

